\title{
ON/OFF VALVE IMPLEMENT OF CONTINUOUS VSC TO A PNEUMATIC CYLINDER*
}

\author{
Akkharaphong EKSIRI**, Tetsuya KIMURA**
}

A pneumatic cylinder system has been difficult in position control due to the nonlinearity of the air, friction on packing seal, and parameter variations. Variable Structure Control (VSC) with a proportion valve has been used to overcome the difficult. However, VSC with an ON/OFF valve is not extensively investigated so far. Therefore, friction compensation using VSC with an ON/OFF valve is proposed in this research. VSC with an ON/OFF valve is designed based on VSC for the proportional one. The experimental results provided the accuracy with $0.1 \mathrm{~mm}$ as same as using a proportional valve with chattering reduction. The results imply that the ON/OFF valve controlled by VSC is a possible replacement of proportional valve for conventional pneumatic cylinder control system.

Key words : Chattering Reduction, On-Off Control, Servo Valve Pneumatic cylinder

\begin{tabular}{|c|c|}
\hline & Nomenclature \\
\hline$m$ & : Payload \\
\hline$x$ & : Displacement of Load mass \\
\hline$v$ & : Velocity \\
\hline$a$ & : Acceleration \\
\hline$L$ & : Stroke length \\
\hline$L_{0}$ & : Initial displacement \\
\hline$F_{P}$ & : Cylinder Force \\
\hline$F_{f}$ & : Friction Force \\
\hline$A_{1}$ & : Piston sectional area \\
\hline$A_{3}$ & : Rod cross-sectional area \\
\hline$A_{2}$ & : Different Area between $A_{1}$ and $A_{3}$ \\
\hline$A_{e i}$ & : Proportional-valve Effective area \\
\hline$\hat{A}_{e i}$ & : ON/OFF-valve Effective area \\
\hline$u$ & : Control action of proportional valve \\
\hline$\hat{u}$ & : Control action of $\mathrm{ON} / \mathrm{OFF}$ valve \\
\hline$V_{i}$ & : Cylinder volume of $\mathrm{i}^{\text {th }}$ chamber \\
\hline$V_{0 i}$ & : Cylinder dead volume of $\mathrm{i}^{\text {th }}$ chamber \\
\hline$Q_{i}$ & : Air Mass flow rate \\
\hline$P_{i}$ & : Absolute pressure of $\mathrm{i}^{\text {th }}$ chamber \\
\hline$P_{a}$ & : Atmosphere pressure \\
\hline$P_{s}$ & : Absolute Air supply pressure \\
\hline$P_{c r}$ & : Critical pressure ratio \\
\hline$R$ & : Gas constant \\
\hline$T$ & : Supply air temperature \\
\hline
\end{tabular}

*Manuscript received August 17, 2007

**Graduate School of Information Science and Control Engineering, Nagaoka University of Technology, JAPAN. aeksiri@stn.nagaokaut.ac.jp

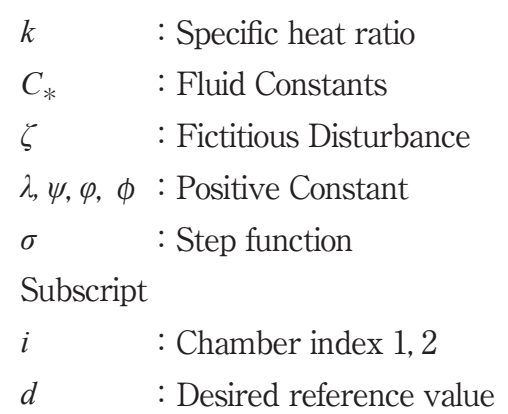

\section{Introduction}

Though, in factory application, a pneumatic cylinder is widely used as an endpoint stop actuator so far, in a field of new robotics such as a disability assisting, it is expected to be controlled as adjustable stroke. However, the position control of a pneumatic cylinder has been difficult due to nonlinearity of the compressibility of air, external disturbances due to friction on packing seals, and so on. Nevertheless, the advantages have been driving researchers to seek the solution for making pneumatic actuators competitive servo systems ${ }^{1)}$.

Many advanced controllers have been applied to control of pneumatic systems and in this research, VariableStructure Control (VSC) is chosen as a controller due to the advantage of low sensitivity to plant parameter variations and disturbances, which eliminates the necessity of exact modeling. Some VSCs of pneumatic cylinders using proportional valveshave been proposed with good performance, e.g. ${ }^{2)}{ }^{4)}$. On the other hand, an ON/OFF valve is widely used in industrial applications because the cost is low and more robust to the environment change. Therefore, a pneumatic cylinder using $\mathrm{ON} / \mathrm{OFF}$ valves is important in the 
viewpoint of usefulness and price advantage in the market. However, VSC with ON/OFF valvesis not extensively investigated so far. For example, the approximation of control laws using Variable Structure Methods in hydraulic servo systems control ${ }^{5)}$ has been evaluated, position control of a pneumatic actuator using ON/OFF solenoid valves by using PID control technique with novel PWM had been proposed for a servo system, and the results have an overshoot when the mass was changed ${ }^{6)}$.

In this paper, a simplified equation of chamber pressure for both cases when the piston extends and retracts is proposed. Based on the model, we design the VSC controller with full state feedback using ON/OFF valve based on proportional valve design method. Closed-loop stability is analyze by Lyapunov's stability method. Chattering phenomenon reduction has been shown explored experimentally. The effectiveness of the proposed method is examined experimentally.

\section{Pneumatic Cylinder Models}

\subsection{Equation of Motion}

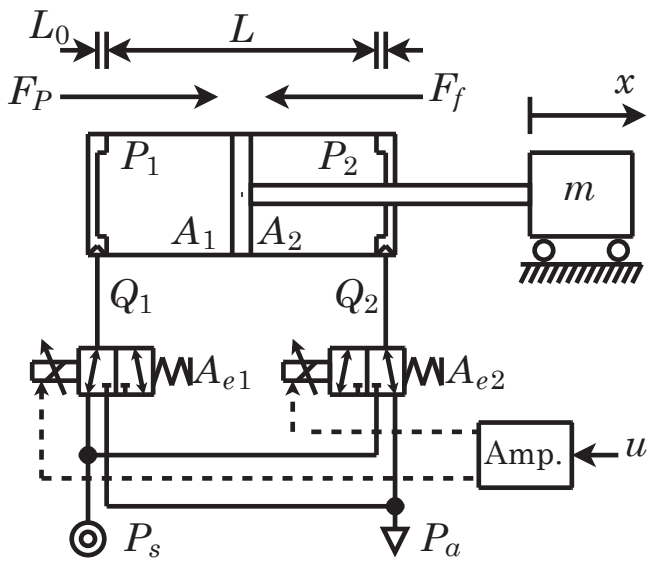

Fig. 1 Pneumatic Cylinder System

Figure 1 shows the schematic of the system: the pneumatic cylinder mounted to two 3/2-way SMCVEF Series valves for Proportional valve and 3/5-way SMCSY3000 Series for ON/OFF valve with a maximum operating pressure (gauge pressure) of $0.5 \mathrm{MPa}$, two pressure sensors for $P_{1}$ and $P_{2}$, and magnetic-type position sensor for $\mathrm{x}$ with resolution of $0.1 \mathrm{~mm}$. The equation of motion of the pneumatic cylinder system can be expressed as :

$$
a=m^{-1}\left(A_{1} P_{1}-A_{2} P_{2}-A_{3} P_{a}-F_{f}\right)
$$

$m$ is Piston+Rod+Loadmass, $x$ is piston position, $F_{f}$ is static friction force, $P_{1,2}$ is the absolute pressure in pneumatic cylinder chamber, $P_{a}$ is the ambient pressure, $A_{1,2}$ is the piston effective area, and $A_{3}$ is the cross-sectional area of the rod.

\subsection{Equation of Chamber Pressure}

The equation of pressure changing insidethe chamber is determined with an ideal gas law, the conservation of mass equation, and the energy equation ${ }^{7}$. Considering the control volume $V$, pressure $P$, temperature $T$, and assumed in isothermal system. For notational simplicity, the two models of the mass flow rate through an valve orifice area $\mathrm{A}_{e i}$ (Choked-flow and Subsonic-flow $)^{3)}$ is denoted by adding the unit-step function of the effective area as shown bellow :

$$
\begin{aligned}
\dot{P}_{i}= & \frac{k}{V_{i}}\left(R T Q_{i}-P_{i} \dot{V}_{i}\right) \\
= & \alpha_{i}\left[K _ { q } \left\{\sigma\left((-1)^{(i+1)} A_{e i}\right) q\left(P_{s}, P_{i}\right)-\sigma\left((-1)^{i} A_{e i}\right)\right.\right. \\
& \left.\left.q\left(P_{i}, P_{a}\right)\right\} A_{e i}+(-1)^{i} P_{i} A_{i} \dot{x}\right], \quad i=1,2
\end{aligned}
$$

where

$$
\begin{aligned}
& \alpha_{i}=\frac{k}{V_{0 i}+A_{i}\left(\frac{L}{2}+(-1)^{i+1} x\right)}, \quad K_{q}=R C_{f} \sqrt{T}, \\
& q\left(P_{u p}, P_{d n}\right)=\left\{\begin{array}{lll}
C_{a} P_{u p} & \text { for } \frac{P_{d n}}{P_{u p}} \leq P_{c r}, \\
C_{b} P_{u p} K_{p} & \text { for } \frac{P_{d n}}{P_{u p}}>P_{c r}
\end{array}\right. \\
& K_{p}=\left(\frac{P_{d n}}{P_{u p}}\right)^{(1 / k)} \sqrt{1-\left(\frac{P_{d n}}{P_{u p}}\right)^{(k-1) / k}} \\
& V_{i}=V_{0 i}+A_{i}\left(\frac{L}{2}+(-1)^{i+1} x\right), \quad \dot{V}_{i}=(-1)^{i+1} A_{i} \dot{x} .
\end{aligned}
$$

$\sigma(\cdot)$ represents the step function, $q(\cdot)$ represents the air flow entering and leaving the chamber. $C_{f}$ is a non dimensional discharge coefficient, $C_{a}$ and $C_{b}$ are fluid constants $^{3)}, P_{u p}$ is upstream pressure, $P_{d n}$ is downstream pressure, and $P_{c r}$ is critical pressure. Subscript $i=1,2$ is the cylinder chamber index, $V_{0 i}$ is the inactive volume, $A_{i}$ is the piston effective area, and $L$ is the piston stroke.

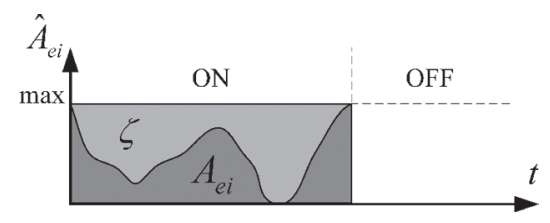

Fig. 2 ON/OFF valve output $\hat{A}_{e i}(t)$ can be represented as Proportional valve output $A_{e i}(t)$ with a fictitious disturbance $\zeta(t)$.

\subsection{Equation of Valve effective area}

The model of effective area Ae of the Proportional valve canbe obtained experimentally. The polynomial curve-fitting method is used to determine the model from experimental data. Resulting in

$$
A_{e i}\left(u_{i}\right)=\left\{\begin{array}{ccc}
a_{1}+a_{2} u_{i}+a_{3} u_{i}^{2} & \text { for } & u>u_{0} \\
0 & \text { for } & u=u_{0} \\
a_{4}+a_{5} u_{i}+a_{6} u_{i}^{2} & \text { for } & u<u_{0}
\end{array}\right.
$$


$a_{1 \sim 6}$ are valve constants, $u_{0}$ is equilibrium voltage. The ON/OFF valve can be modeled as :

$$
\hat{A}_{e i}=\left\{\begin{array}{ccc}
A_{e i}^{+} & \text {for } & u>0 \\
0 & \text { for } & u=0 \\
A_{e i}^{-} & \text {for } & u<0
\end{array}\right.
$$

where $\hat{A}_{e i}$ is the effective area of the ON/OFF valve. The relation between $A_{e}\left(u_{i}\right)$ and $\hat{A}_{e}\left(u_{i}\right)$ can be expressed as :

$$
\hat{A}_{e i}\left(u_{i}\right)=A_{e i}\left(u_{i}\right)+\zeta(t)
$$

where $\zeta(t)$ is a fictitious disturbance as shown in Fig. 2 and this is represented as an additive disturbance in Fig. 3. Note that $\zeta(t)$ is only used in stability analysis in Section 4, and not used in control.

\section{Variable Structure Controller Design}

\subsection{VSC design with Proportional Valve}

By following the design method in Bonchis's), we have designed the control law for the case when piston extends and retracts using Eqs. (1), (2), and (7). From equation ofmotion as shownin Eq. (1), denoting: velocity $\dot{x}=v$, acceleration $\dot{v}=a$, then

$$
\dot{a}=\frac{1}{m}\left(A_{1} \dot{P}_{1}-A_{2} \dot{P}_{2}\right)
$$

Substituting the equation of chamber pressure Eq. (2) into Eq. (10), we can rewrite Eq. (10) in nonlinear state equation as:

$$
\dot{a}=f\left(x, v, P_{1}, P_{2}\right)+b\left(x, P_{1}, P_{2}, A_{e i}\right) \cdot A_{e}(u)
$$

where

$$
\begin{aligned}
f(\cdot)= & -\frac{v}{m}\left(\alpha_{1} A_{1}^{2} P_{1}+\alpha_{2} A_{2}^{2} P_{2}\right) \\
b(\cdot)= & \frac{K_{q}}{m}\left[\alpha_{1} A_{1}\left\{\sigma\left(A_{e}\right) q\left(P_{s}, P_{1}\right)-\sigma\left(-A_{e}\right) q\left(P_{1}, P_{a}\right)\right\}\right. \\
& -\alpha_{2} A_{2}\left\{\sigma\left(-A_{e}\right) q\left(P_{s}, P_{2}\right)-\sigma\left(A_{e}\right) q\left(P_{2}, P_{a}\right)\right\} . \text { (13) }
\end{aligned}
$$

where $A_{e}(u)=A_{e 1}(u)=-A_{e 2}(u)$. Now, define $e_{x}=x_{d}-x$ be the error in the piston position $x$ with desired value representedby subscript $d$. For the system Eq. (11) the switching surface $s=0$ of VSC is defined ${ }^{10)}{ }^{11)}$ :

$$
s=\left(\frac{d}{d t}+\lambda\right)^{n-1} \cdot e_{x}
$$

where $\lambda$ is a strictly positive constant that effects to desired rising time of the closed-loop system, $n=3$ for our third order system. Then Eq. (14) becomes :

$$
\begin{aligned}
& s=e_{a}+2 \lambda e_{v}+\lambda^{2} e_{x} \\
& \dot{s}=\dot{e}_{a}+2 \lambda e_{a}+\lambda^{2} e_{v}
\end{aligned}
$$

where $e_{v}=\dot{e}_{x}=v_{d}-v$ represents the tracking error of the velocity and $e_{a}$ represents the acceleration error and these are obtained by a numerical differentiation method of position data. The control action can be determined from the condition of $\dot{s}=0$, taking Eqs. (1) and (11) into Eq. (16), resulting in

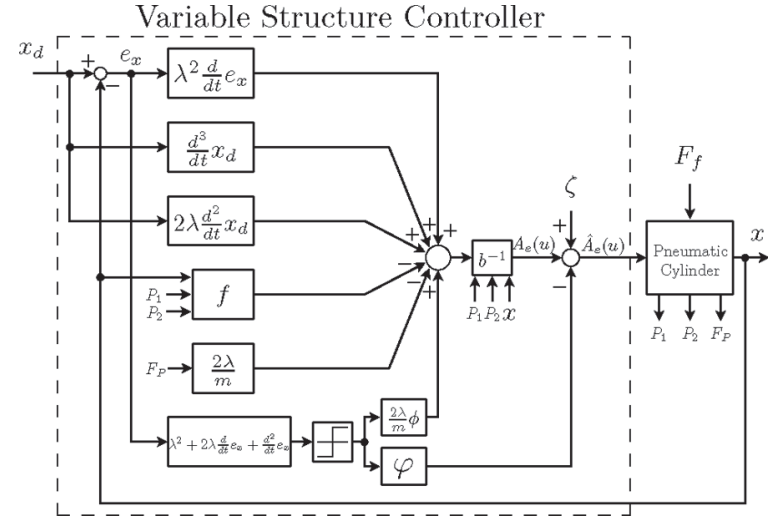

Fig. 3 Variable Structure Controller Block Diagram with $\mathrm{ON} / \mathrm{OFF}$ valve

$$
\begin{aligned}
& A_{e}(u)=b^{-1}\left(-f+\dot{a}_{d}+2 \lambda a_{d}+\lambda^{2} e_{v}-\frac{2 \lambda F_{p}}{m}\right)+b^{-1} \frac{2 \lambda}{m} F_{f} \\
& F_{p}=A_{1} P_{1}-A_{2} P_{2}-A_{3} P_{a}
\end{aligned}
$$

Note that $A_{e}$ is invertible as shown in Eq. (7). To eliminate the effect of the static friction $F_{f}$, it can be replaced by signum function of switching surface (14) as shown bellows :

$$
\begin{aligned}
& F_{f} \rightarrow \phi \operatorname{sgn}(s) \\
& \phi>\left|F_{f}\right|>0
\end{aligned}
$$

where, $\phi$ ispositive constant of the friction boundary. Now, we obtained the control action $u$ as :

$$
u=A_{e}^{-1}\left(u_{e q}+b^{-1} \frac{2 \lambda}{m} \phi \operatorname{sgn}(s)\right)
$$

where

$$
u_{e q}=b^{-1}\left(-f+\dot{a}_{d}+2 \lambda a_{d}+\lambda^{2} e_{v}-\frac{2 \lambda F_{p}}{m}\right)
$$

\subsection{VSC design with ON/OFF valve}

To design the controller by using ON/OFF valve based on Proportional one, the control action in Eq. (11) will be replace by Eq. (9) and then Eq. (11) becomes :

$$
\dot{a}=f+b \cdot\left(A_{e}(u)+\zeta\right)
$$

The VSC (21) becomes :

$$
u=A_{e}^{-1}\left(u_{e q}+b^{-1} \frac{2 \lambda}{m} \phi \operatorname{sgn}(s)-\varphi \operatorname{sgn}(s)\right)
$$

where

$$
\varphi>|\zeta|>0
$$

The control action for the pneumatic cylinder with ON/ OFF valve becomes :

$$
\hat{u}=\left\{\begin{array}{ccc}
u^{+} & \text {for } & u>0 \\
0 & \text { for } & u=0 \\
u^{-} & \text {for } & u<0
\end{array}\right.
$$

$\hat{u}$ is the control signal for the ON/OFF-type of valve, $u^{+}$is fully control action voltage when the piston extends, and $u^{-}$ when the piston retracts. Figure. 3 shows the Variable Structure Controller Block Diagram. 


\section{Stability Analysis of VSC with ON/OFF-valve}

To analyze the stability of close-loop control, by using the Lyapunov's function $^{8)}$ 9) candidate,

$$
v=0.5 s^{2}
$$

a stability condition is given by

$$
\dot{v}=s \dot{s} \leq 0 \text {. }
$$

Taking Eqs. (1), (11), and (24) into Eq. (16) and assumed that the desired value is step-type function. Thetreatment of the another functions of desired value are similar. Now, the first time derivative of Eq. (27) can be obtained as

$$
\dot{v}=s\left(-\frac{2 \lambda}{m}\left(\phi \operatorname{sgn}(s)-F_{f}\right)+b(\varphi \operatorname{sgn}(s)-\zeta)\right)
$$

Taking Eqs. (13), (15), (20), and (25) into consideration, the condition of (28) is satisfied if

$$
\left|\frac{2 \lambda}{m}\left(\phi \operatorname{sgn}(s)-F_{f}\right)\right| \geq|b(\varphi \operatorname{sgn}(s)-\zeta)| \geq 0 .
$$

Note that, inequality (30) with $\zeta=\varphi=0$, which implies that the proportional valvecase makes the inequality (30) always be satisfied, is identical to the stability condition of VSC with a proportional valve to eliminates the effect of the static friction ${ }^{8)}$.

\section{Experimental Results}

\subsection{VSC with Proportional Valve}

The valves in Table.1 are used in the experiment.

Table 1 Proportional VS ON/OFF Valve

\begin{tabular}{l|c|c|c}
\hline \multicolumn{1}{c|}{ Valve } & Ae $\left[\mathrm{mm}^{2}\right]$ & $\mathrm{RT}^{*}[\mathrm{~ms}]$ & Price $[\mathrm{USD}]$ \\
\hline \hline Proportional & 1.0 & 30 & 180 \\
\hline ON/OFF & 1.0 & 10 & 80 \\
\hline
\end{tabular}

* RT : Response Time in catalog

\subsubsection{Control Parameter Determination}

Two major parameters $\lambda$ and $\phi$ of the controller in Eq. (21) are determined under the stability condition of Eq. (30) with $\varphi=\zeta=0$. The value of $\phi=30$ canbe determined by expression of Eq. (20), with experimental consideration of $F_{f}$. The value of $\lambda=50$ can be obtained by trial and error technique in tuning phase that depends on desire rising time of the closed-loop system. The reference position of closed-loop system control is a Square-wave with $0.05 \mathrm{~m}$ amplitude and $0.25 \mathrm{~Hz}$ frequency and Table 2 shows the experimental parameters. The designed controller (24) provided position response curve with chattering phenomenon as shown in Fig. 4. The chattering phenomenon ${ }^{10)}$ is in bound 20 (see Fig. 4 bottom), and this value of switching surface will be discuss in next section.

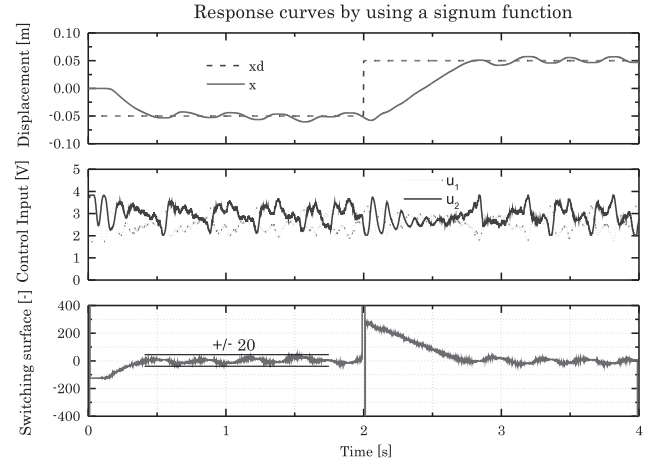

Fig. 4 Position Response using Proportional-valves without chattering reduction

\subsubsection{Chattering Reduction}

In order to reducethe chattering phenomenon, the signum function in control action Eq. (24) can be replace by one of four functions as follows :

- Smoothing Function

$$
\operatorname{sgn}(s) \rightarrow \frac{s}{|s|+\delta}
$$

- Dead-Zone Function

$$
\operatorname{sgn}(s) \rightarrow\left\{\begin{array}{ccc}
+1, & \text { for } & s>\delta \\
0, & \text { for } & -\delta \leq s \leq \delta \\
-1, & \text { for } & s<-\delta
\end{array}\right.
$$

- Hyperbolic Tangent Function

$$
\operatorname{sgn}(s) \rightarrow \tanh \left(\frac{s}{\delta}\right)
$$

Table 2 Experimental Parameters

\begin{tabular}{c|c|c}
\hline Parameters & Value & Unit \\
\hline \hline $\mathrm{A}_{1}$ & 314 & $\mathrm{~mm}^{2}$ \\
\hline $\mathrm{A}_{2}$ & 235 & $\mathrm{~mm}^{2}$ \\
\hline $\mathrm{A}_{3}$ & 78.5 & $\mathrm{~mm} \mathbf{m}^{2}$ \\
\hline $\mathrm{m}$ & 3.0 & $\mathrm{~kg}$ \\
\hline $\mathrm{L}$ & 0.2 & $\mathrm{~m}$ \\
\hline $\mathrm{V}_{01}$ & 314 & $\mathrm{~mm}^{3}$ \\
\hline $\mathrm{V}_{02}$ & 235 & $\mathrm{~mm}$ \\
\hline $\mathrm{R}$ & 287 & $\mathrm{~N} \cdot \mathrm{m} / \mathrm{kg} \cdot \mathrm{K}$ \\
\hline $\mathrm{C}_{f}$ & 0.23 & - \\
\hline $\mathrm{C}_{a}$ & 0.04 & $\sqrt{1 / K}$ \\
\hline $\mathrm{C}_{b}$ & 0.16 & $\sqrt{1 / K}$ \\
\hline $\mathrm{T}$ & 293 & $\mathrm{~K}$ \\
\hline $\mathrm{k}$ & 1.4 & - \\
\hline $\mathrm{P}_{a}$ & 0.1 & $\mathrm{MPa}$ \\
\hline $\mathrm{P}_{s}$ & 0.6 & $\mathrm{MPa}$ \\
\hline $\mathrm{P}_{c r}$ & 0.5 & - \\
\hline
\end{tabular}




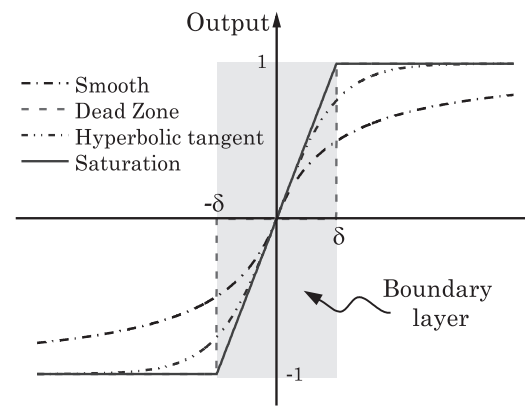

Fig. 5 Chattering Reduction Methods with same $\delta$

- Saturation Function

$$
\operatorname{sgn}(s) \rightarrow \operatorname{sat}\left(\frac{s}{\delta}\right)=\left\{\begin{array}{ccc}
+1, & \text { for } & s>\delta \\
\frac{s}{\delta}, & \text { for } & -\delta \leq s \leq \delta \\
-1, & \text { for } & s<-\delta
\end{array}\right.
$$

Figure 5 shows the output of above chattering reduction methods with same value of $\delta$. Now, let we investigate the performance of each chattering reduction method using $\delta$ is 20, and Fig. 6 shows the results.

Table 3 Comparison of Chattering Reduction Methods

\begin{tabular}{c|c|c}
\hline Methods & Settling time $[\mathrm{s}]$ & error $[\mathrm{mm}]$ \\
\hline \hline Smooth Function & 0.36 & 0.4 \\
\hline Dead-Zone Function & 0.31 & 4.0 \\
\hline Hyperbolic Tangent & 0.39 & 0.2 \\
\hline Saturation Function & 0.30 & 0.1 \\
\hline
\end{tabular}

Observing that, all chattering reduction methods canreduce the chattering phenomen on. The differences in position accuracy can be discussed as follows : the position response curve susing Smooth and Hyperbolic tangent function when the switching surface is out of boundary layer neighboring the switching surface ${ }^{10)}$ are less than one (see Fig. 5), that have been an effect to the control action is lower than properly. For the dead-zone and saturation function has no
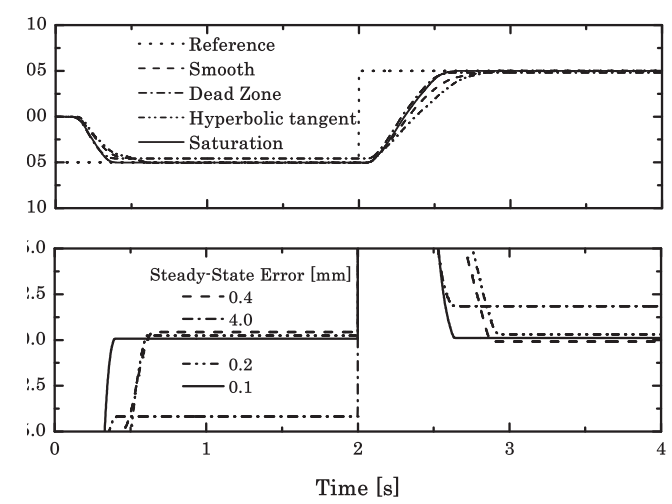

Fig. 6 Position Response using Proportional-valves with chattering reduction such an effect as described above but the dead-zone function is zero when $\mathrm{s}$ is in the bound of switching surface that also degrade the control action and provide the largest steadystate error. The comparison of VSC with chattering reduction methods are shownin Table 3. Consequently, the VSC with saturation function is an appropriat eimproving method to reduce the chattering phenomenon since its provided of lowerrise time, smaller error, and smoother motion than other three methods.

\subsection{VSC with ON/OFF Valve}

\subsubsection{Control Parameter Determination}

Three major parameters of the controllerin Eq. (24) are also determined under the stability condition of Eq. (30) with the same plant parameters as VSC with proportional valve with chattering reduction method of saturation function. For $\lambda$ and $\varphi$ are same as VSC with proportional valve, and $\varphi=$ 1.01 can be determinedby expression (25) with consideration of maximum value of the effective area of the valve with 1.0 $\mathrm{mm}^{2}$

\subsubsection{Comparison to Proportional Valve}

The experimentals with ON/OFFvalves are carried out by using the same reference position of Square wave as VSC with proportional valve in Fig. 6. The Displacement [m] Displacement [m] result are shown in Fig. 7.

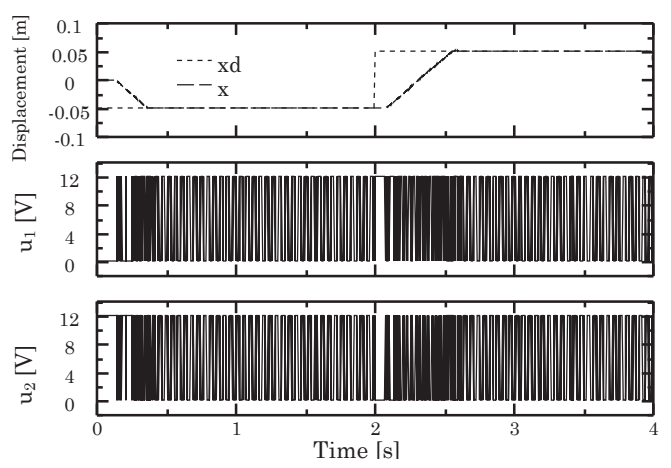

Fig. 7 Position Response using ON/OFF valves with chattering reduction of saturation function

VSC with ON/OFF valve provided good performance of position controls same as the using of proportional valves. The comparison of both results between proportional and ON/OFF valves in detail are shown in Fig. 8. Observing that, the VSC with both type of valves provided almost the same settling time and the steady-state error is $0.1 \mathrm{~mm}$ for VSC with proportional valve, $0.6 \mathrm{~mm}$ for $\mathrm{VSC}$ with $\mathrm{ON} / \mathrm{OFF}$ valve.

\subsubsection{Robustness Investigation}

The robustness investigation are carried out as follow. Figure 9 above $(m=3.0 \mathrm{~kg})$ shows the experimental results 

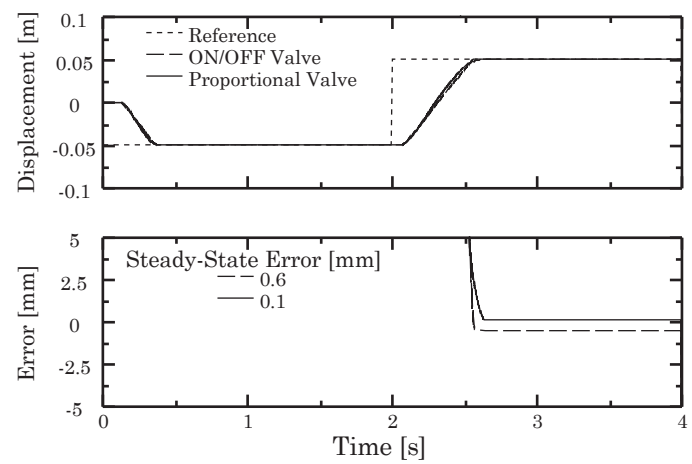

Fig. 8 VSC with Proportional and ON/OFF valve with chattering reduction of saturation function
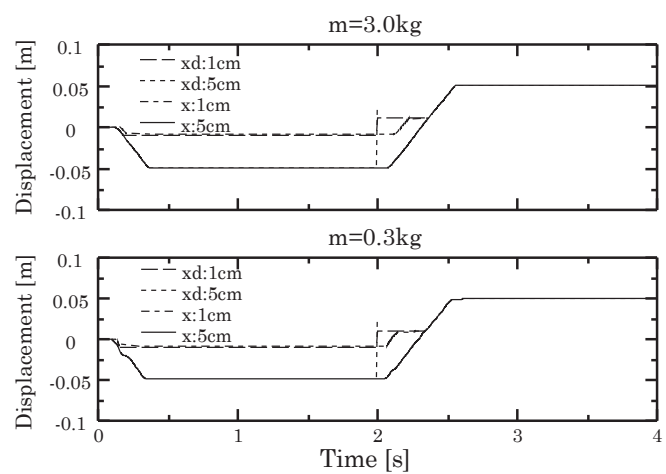

Fig. 9 VSC with ON/OFF valve Robustness In vestigation in Variation of references input and payload

with square-wave position reference signal of $0.05 \mathrm{~m}, 0.01 \mathrm{~m}$ amplitude and $0.25 \mathrm{~Hz}$ frequency. Dash-dot line represents the position response of $0.01 \mathrm{~m}$ reference input and Dashdot-dot line represent the position response of $0.05 \mathrm{~m}$ reference input. For both of position response curves, the VSC with $\mathrm{ON} / \mathrm{OFF}$ valves still provided good performance in position control, and the steady-state error is in bound of the allowable to lerence ( $2 \%$ of reference) as shown in Table 4

Table 4 Comparisonof Variation of Reference Input

\begin{tabular}{c|c}
\hline Reference Position $[\mathrm{mm}]$ & Steady-state error $[\mathrm{mm}]$ \\
\hline \hline 10 & 0.1 \\
\hline 50 & 0.6 \\
\hline
\end{tabular}

And also the robustness of designed VSC in payload and input variation are investigated. The nominal mass of the system (piston, rod, linear slide, and payload) is $m=3 \mathrm{~kg}$. The remove of the payload decreased the mass by 10 times to $m=0.3 \mathrm{~kg}$. Figure 9 bellow $(m=0.3 \mathrm{~kg})$ shows position response with the same reference signal as in Fig. 9 above $(m=0.3 \mathrm{~kg})$. Observing the responses, the designed VSC provided similar rise time to nominal one and the overshoot phenomenon $^{6)}$ is not appeared. Even though the steadystate error of VSC wiht ON/OFF valve is biger than proportional valve, the VSC with ON/OFF valve having a useful on price advantage (Table. 1) .

\section{Conclusion}

The Variable Structure Control (VSC) with ON /OFF valve for eliminate the effect of the static friction have been proposed based on VSC with Proportional valve. A fictitious disturbance in control action have been also proposed to examine rebustness theoretically as in Eq. (30). Experimentally identified model with ordinary equations has been carried out. By using the models, the VSC with ON/OFF valve has been designed based on proportional valve method. Closed-loop stability analysis of VSC with ON/OFF valve using Lyapunov's function has been carried out. The chattering phenomenon reduction by using the saturation function has been introduced by comparing to other methods experimentally. The comparison of VSC using proportional and ON/OFF valve with the same of control parameters have been carried out and both have comparable performances. The robustness of designed VSC with $\mathrm{ON} / \mathrm{OFF}$ valve for the variation of reference input and payload have been investigated and experimental results provided similar response to nominal ones, which implies good robustness of the proposed method. Therefor, by using the proposed VSC with $\mathrm{ON} / \mathrm{OFF}$ valve, the $\mathrm{ON} / \mathrm{OFF}$ valves can be a replacement of the proportional valves in pneumatic cylinder systems. A further issue is detailed interpretation of the robustness condition in Eq. (30).

\section{References}

1 ) FulinXiang, JanWikander, Block-oriented approxi-mate feedback linearization for control of pneumatic actuator system, Control Engineering Practice 12, pp. 387-399, (2004)

2 ) Twu-Chung Su, Chun-Yen Euo, Variable structure control of a rodless pneumatic servo actuator with discontinuous sliding surfaces, Proceedings of the 2000 American Control Conference, Vol. 3, pp. 1617-1621, (2000)

3 ) Ben-Dov, D., and Salcudean, S.E., A Force-Controlled Pneumatic Actuator, IEEE Transactions on Robotics and Automation, Vol. 11, No. 6, pp. 906-911, (1995)

4 ) Toshiro Noritsugu, Tsutomu Wada, and Masayuki Shigeyoshi, Compliane Control of a Pneumatic Robot with Improved Sliding Mode Control Method, Transaction of SICE, Vol. 27, No. 1, pp. 77-84, (1991), (in 
Japanese)

5 ) A. Bonchis, P.I. Corke, D.C. Rye, Q.P. Ha, Variable structure methods in hydraulic servo systems control, Automatica, Vol. 37, pp. 589-595, (2001)

6 ) Robert B. van Varseveld and Gray M. Bone, Accurate Position Control of a Pneumatic ActuatorUsing On/Off Solenoid Valves, IEEE/ASME Transactions on Mechatronics, Vol. 2, No. 3, pp. 195-204, Sep., (1997)

7 ) Hullender, D.A., and Woods, R.L., Modeling of Fluid Control Components, Proceedings of the First Conference on Fluid Control and Measurement, FLUCOME '85, Tokio, London : Pergam on Press, (1985)

8 ) Q.P. Ha, A. Bonchis, D.C. Rye, and H.F. Durrant-
Whyte, Variable Structure Systems Approach to Friction Estimation and Compensation, IEEE International Conference on Robotics and Automation, Vol. 4, pp. 3543-3548, (2000)

9 ) Q.P. Ha, Sliding performance enhancement with fuzzy tuning, IEE Electronics Letters, Vol. 33, No. 16, pp. 1421-1423, July, (1997)

10) Jean-Jacques E. Slotine, and Weiping Li, Applied Nonlinear Control, Prentice-Hall International Editions, (1991)

11) Asif Sabanovic, Leonid M. Fridman, and Sarah Spurgeon, Variable Structure Systems : from Principles to Implementation, IEE Control Series 66, (2004) 